\title{
The deformations of symplectic structures by moment maps
}

\author{
Tomoya Nakamura * \\ email: x-haze@ruri.waseda.jp
}

May 10, 2016

\begin{abstract}
We study deformations of symplectic structures on a smooth manifold $M$ via the quasi-Poisson theory. By a fact, we can deform a given symplectic structure $\omega$ to a new symplectic structure $\omega_{t}$ parametrized by some element $t$ in $\Lambda^{2} \mathfrak{g}$, where $\mathfrak{g}$ is the Lie algebra of a Lie group $G$. Moreover, we can get a lot of concrete examples for the deformations of symplectic structures on the complex projective space and the complex Grassmannian.
\end{abstract}

\section{Introduction}

In the context of symplectic geometry, deformation-equivalence assumptions and conditions are often appeared, for example, in the statement of Moser's theorem [9] and Donaldson's four-six conjecture [8]. However, it seems that a method of constructing deformation-equivalent symplectic structures specifically is not well known. In this paper, we construct a method of producing new symplectic structures deformation-equivalent to a given symplectic structure. Our approach to deformations of symplectic structures is to use quasi-Poisson theory which was introduced by Alekseev and KosmannSchwarzbach [1, and this approach is carried out by using the fact that a moment map for a symplectic-Hamiltonian action $\sigma$ is also a moment map for a quasi-Poisson action $\sigma$. The former moment map satisfies conditions for only one symplectic structure, whereas the latter does conditions for a family of quasi-Poisson structures parametrized by elements in $\Lambda^{2} \mathfrak{g}$. From

\footnotetext{
* Department of Mathematics, Waseda University, 3-4-1, Okubo, Shinjuku-ku, Tokyo, Japan
} 
here we call these elements twists. Regarding the quasi-Poisson structure induced by a symplectic structure as that with twist 0 , which is denoted by $\pi_{0}$, we can find different quasi-Poisson structures $\pi_{t}$ which induce symplectic structures $\omega_{t}$ by the choice of "good" twists $t$. The quasi-Poisson structure inducing a symplectic structure must be a nondegenerate Poisson structure. We describe the conditions for the quasi-Poisson structure with a twist $t$ to be a nondegenerate Poisson structure. Our method of using the family of quasi-Poisson structures is one of interesting geometry frameworks (See [1]).

From here, we explain briefly the difference among moment maps for symplectic- and quasi-Poisson-Hamiltonian actions, and equivariant moment maps for Poisson actions on a smooth manifold (In Poisson geometry, nonequivariant moment maps for Poisson action can be defined [4], 5].).

(I) Symplectic-Hamiltonian actions

In symplectic geometry, a moment map $\mu: M \rightarrow \mathfrak{g}^{*}$ for a symplectic action $\sigma$ of a Lie group $G$ on a symplectic manifold $(M, \omega)$ is defined with two conditions: one is for the symplectic structure $\omega$,

$$
d \mu^{X}=\iota_{X_{M}} \omega(X \in \mathfrak{g}) .
$$

Here $\mu^{X}(p):=<\mu(p), X>$ and $X_{M}$ is a vector field on $M$ defined by

$$
X_{M, p}:=\left.\frac{d}{d t} \sigma_{\exp t X}(p)\right|_{t=0}
$$

for $p$ in $M$. The other is the $G$-equivariance condition with respect to the action $\sigma$ on $M$ and the coadjoint action $\mathrm{Ad}^{*}$ on $\mathfrak{g}^{*}$,

$$
\mu \circ \sigma_{g}=\operatorname{Ad}_{g}^{*} \circ \mu
$$

for all $g$ in $G$. In this paper, we call symplectic actions with moment maps symplectic-Hamiltonian actions to distinguish it from other actions with moment maps.

(II) Poisson actions with equivariant moment maps

A Poisson Lie group, which was introduced by Drinfel'd [3], is a Lie group with a Poisson structure $\pi$ compatible with the group structure. Namely, the structure $\pi$ satisfies

$$
\pi_{g h}=L_{g *} \pi_{h}+R_{h *} \pi_{g}
$$

for any $g$ and $h$ in $G$, where $L_{g}$ and $R_{h}$ are the left and right translations in $G$ by $g$ and $h$, respectively. Such a structure is called multiplicative. Then the simply connected Lie group $G^{*}$ called the dual Poisson Lie group is 
obtained uniquely from a Poisson Lie group $(G, \pi)$ and a local action of $G$ on $G^{*}$ is defined naturally. We call a multiplicative Poisson structure $\pi$ on $G$ complete if the action is global. Then $(G, \pi)$ is called a complete Poisson Lie group. An equivariant moment map $\mu: M \rightarrow G^{*}$ for a Poisson action $\sigma$ of a complete Poisson Lie group $(G, \pi)$ on a Poisson manifold $(M, \pi)$ is a generalization of a moment map for a symplectic-Hamiltonian action on a symplectic manifold, which was given by $\mathrm{Lu}$ in [4].

(III) Quasi-Poisson-Hamiltonian actions

Quasi-Poisson theory, which was originated with [1] by Alekseev and Kosmann-Schwarzbach, is a generalization of Poisson theory with Poison actions. In quasi-Poisson geometry, quasi-triples $(D, G, \mathfrak{h})$ and its infinitesimal version, Manin quasi-triples $(\mathfrak{d}, \mathfrak{g}, \mathfrak{h})$, play important roles. A quasitriple $(D, G, \mathfrak{h})$ defines a quasi-Poisson Lie group $G_{D}^{\mathfrak{h}}$ and we can obtain the

notion of a quasi-Poisson action of such a quasi-Poisson Lie group $G_{D}^{\mathfrak{h}}$. A moment map $\mu$ for the action is a map from $M$ into $D / G$ and satisfies a condition not for one quasi-Poisson structure but for a family of quasi-Poisson structures parametrized by elements in $\Lambda^{2} \mathfrak{g}$. An equivariant moment map for a Poisson action in (II) is an example of a moment map for a quasiPoisson-Hamiltonian action. In this paper, we use the moment map theory for quasi-Poisson actions to deform symplectic structures on a smooth manifold.

This paper is constructed as follows. It is contents of Section 2 to review the moment map theory for quasi-Poisson actions. In Section 3, we describe a deformation method of symplectic structures on a smooth manifold via the quasi-Poisson theory. This method is the subject in this paper. Theorem 3.1 gives a condition for a twist to deform a symplectic structure to a new one. In addition, Theorem 3.2 gives a sufficient condition for a twist to satisfy the assumption of Theorem 3.1. In Section 4, we introduce concrete examples for deformations of symplectic structures. We give deformations of the Fubini-Study and the Kirillov-Kostant forms on $\mathbb{C P}^{n}$ and the complex Grassmannian, respectively.

\section{Moment maps for quasi-Poisson actions on quasi- Poisson manifolds}

In this section, we shall recall the quasi-Poisson theory [1]. We start with the definition of quasi-Poisson Lie groups, which is a generalization of Poisson Lie groups. 
Definition 1. Let $G$ be a Lie group with the Lie algebra $\mathfrak{g}$. Then a pair $(\pi, \varphi)$ is a quasi-Poisson structure on $G$ if a multiplicative 2-vector field $\pi$ on $G$ and an element $\varphi$ of $\Lambda^{3} \mathfrak{g}$ satisfy

$$
\begin{aligned}
& \frac{1}{2}[\pi, \pi]=\varphi^{R}-\varphi^{L}, \\
& {\left[\pi, \varphi^{L}\right]=\left[\pi, \varphi^{R}\right]=0,}
\end{aligned}
$$

where the bracket $[\cdot, \cdot]$ is the Schouten bracket on the multi-vector fields on $G$, and $\varphi^{L}$ and $\varphi^{R}$ denote the left and right invariant 2-vector fields on $G$ with value $\varphi$ at e respectively. A triple $(G, \pi, \varphi)$ is called a quasi-Poisson Lie group.

Remark 1. In a quasi-Poisson structure $(\pi, \varphi)$ on $G$, the 2-vector field $\pi$ is a multiplicative Poisson structure if $\varphi=0$. Namely, $(G, \pi)$ is a Poisson Lie group.

We use a "quasi-triple" to obtain a quasi-Poisson Lie group. To define a quasi-triple, we describe its infinitesimal version, a Manin quasi-triple.

Definition 2. Let $\mathfrak{d}$ be a $2 n$-dimensional Lie algebra with an invariant nondegenerate symmetric bilinear form of signature $(n, n)$, which is denoted by $(\cdot \mid \cdot)$. Let $\mathfrak{g}$ be an $n$-dimensional Lie subalgebra of $\mathfrak{d}$ and $\mathfrak{h}$ be an $n$ dimensional vector subspace of $\mathfrak{d}$. Then a triple $(\mathfrak{d}, \mathfrak{g}, \mathfrak{h})$ is a Manin quasitriple if $\mathfrak{g}$ is a maximal isotropic subspace with respect to $(\cdot \mid \cdot)$ and $\mathfrak{h}$ is an isotropic complement subspace of $\mathfrak{g}$ in $\mathfrak{d}$.

Remark 2. For a given Lie algebra $\mathfrak{d}$ and a Lie subalgebra $\mathfrak{g}$ of $\mathfrak{d}$, a choice of an isotropic complement subspace $\mathfrak{h}$ of $\mathfrak{g}$ in $\mathfrak{d}$ is not unique.

A Manin quasi-triple $(\mathfrak{d}, \mathfrak{g}, \mathfrak{h})$ defines the decomposition $\mathfrak{d}=\mathfrak{g} \oplus \mathfrak{h}$. Then the linear isomorphism

$$
j: \mathfrak{g}^{*} \rightarrow \mathfrak{h},(j(\xi) \mid x):=<\xi, x>\left(\xi \in \mathfrak{g}^{*}, x \in \mathfrak{g}\right)
$$

is determined by the decomposition. We denote the projections from $\mathfrak{d}=$ $\mathfrak{g} \oplus \mathfrak{h}$ to $\mathfrak{g}$ and $\mathfrak{h}$ by $p_{\mathfrak{g}}$ and $p_{\mathfrak{h}}$ respectively. We introduce an element $\varphi_{\mathfrak{h}}$ in $\Lambda^{3} \mathfrak{g}$ which is defined by the map from $\Lambda^{2} \mathfrak{g}^{*}$ to $\mathfrak{g}$, denoted by the same letter,

$$
\varphi_{\mathfrak{h}}(\xi, \eta)=p_{\mathfrak{g}}([j(\xi), j(\eta)]),
$$

for any $\xi, \eta$ in $\mathfrak{g}^{*}$. We define the linear map $F_{\mathfrak{h}}: \mathfrak{g} \rightarrow \Lambda^{2} \mathfrak{g}$ by setting

$$
F_{\mathfrak{h}}^{*}(\xi, \eta)=j^{-1}\left(p_{\mathfrak{h}}([j(\xi), j(\eta)])\right)
$$


for any $\xi, \eta$ in $\mathfrak{g}^{*}$, where $F_{\mathfrak{h}}^{*}: \Lambda^{2} \mathfrak{g}^{*} \rightarrow \mathfrak{g}^{*}$ is the dual map of $F_{\mathfrak{h}}$. These elements will be used later to define a quasi-Poisson structure and a quasiPoisson action respectively.

Next we define a quasi-triple $(D, G, \mathfrak{h})$ and construct a quasi-Poisson structure on $G$ using $(D, G, \mathfrak{h})$.

Definition 3. Let $D$ be a connected Lie group with a bi-invariant scalar product with the Lie algebra $\mathfrak{d}$ and $G$ be a connected closed Lie subgroup of $D$ with the Lie algebra $\mathfrak{g}$. Let $\mathfrak{h}$ be a vector subspace of $\mathfrak{d}$. Then a triple $(D, G, \mathfrak{h})$ is a quasi-triple if $(\mathfrak{d}, \mathfrak{g}, \mathfrak{h})$ is a Manin quasi-triple.

A method of constructing a quasi-Poisson structure by a quasi-triple is as follows. Let $(D, G, \mathfrak{h})$ be a quasi-triple with a Manin quasi-triple $(\mathfrak{d}, \mathfrak{g}, \mathfrak{h})$. Using the inverse $j^{-1}: \mathfrak{h} \rightarrow \mathfrak{g}^{*}$ of the linear isomorphism (5), we identify $\mathfrak{d}$ with $\mathfrak{g} \oplus \mathfrak{g}^{*}$. Consider the map

$$
r_{\mathfrak{h}}: \mathfrak{d}^{*} \rightarrow \mathfrak{d}, \xi+X \mapsto \xi
$$

for any $\xi$ in $\mathfrak{g}^{*}$ and $X$ in $\mathfrak{g}$. This map defines an element $r_{\mathfrak{h}} \in \mathfrak{d} \otimes \mathfrak{d}$ which we denote by the same letter. We set

$$
\pi_{D}^{\mathfrak{h}}:=r_{\mathfrak{h}}^{L}-r_{\mathfrak{h}}^{R},
$$

where $r_{\mathfrak{h}}^{L}$ and $r_{\mathfrak{h}}^{R}$ is denoted as the left and right invariant 2-tensors on $D$ with value $r_{\mathfrak{h}}$ at the identity element $e$ in $D$ respectively, and we can see that it is a multiplicative 2-vector field on $D$. Furthermore, the 2 -vector field $\pi_{D}^{\mathfrak{h}}$ and the element $\varphi_{\mathfrak{h}}$ defined by (6) satisfy (3i) and (4). We set

$$
\pi_{G, g}^{\mathfrak{h}}:=\pi_{D, g}^{\mathfrak{h}}
$$

for any $g$ in $G$. Then we can see that $\pi_{G}^{\mathfrak{h}}$ is well-defined and that $\pi_{G}^{\mathfrak{h}}$ is a multiplicative 2-vector field on $G$. Moreover, $\pi_{G}^{\mathfrak{h}}$ and $\varphi_{\mathfrak{h}}$ satisfy (3) and (4). Therefore $\left(G, \pi_{G}^{\mathfrak{h}}, \varphi_{\mathfrak{h}}\right)$ is a quasi-Poisson Lie group. We sometimes denote a Lie group with such a structure by $G_{D}^{\mathfrak{h}}$.

From here, we consider only connected quasi-Poisson Lie group $G_{D}^{\mathfrak{h}}$ defined as above by a quasi-triple $(D, G, \mathfrak{h})$. For a smooth manifold $M$ with a 2-vector field $\pi_{M}$, a quasi-Poisson action is defined as follows. It is a generalization of Poisson actions of connected Poisson Lie groups [6].

Definition 4. Let $G_{D}^{\mathfrak{h}}$ be a connected quasi-Poisson Lie group acting on a smooth manifold $M$ with a 2-vector field $\pi_{M}$. The action $\sigma$ of $G$ on $M$ is a 
quasi-Poisson action if for each $X$ in $\mathfrak{g}$,

$$
\begin{aligned}
\frac{1}{2}\left[\pi_{M}, \pi_{M}\right] & =\left(\varphi_{\mathfrak{h}}\right)_{M}, \\
\mathfrak{L}_{X_{M}} \pi_{M} & =F_{\mathfrak{h}}(X)_{M},
\end{aligned}
$$

where $x_{M}$ is a fundamental multi-vector field for any $x$ in $\wedge^{*} \mathfrak{g}$. Here $F_{\mathfrak{h}}$ is the dual of the map (7). Then a 2-vector field $\pi_{M}$ is called a quasi-Poisson $G_{D}^{\mathfrak{h}}$-structure on $M$ and $\left(M, \pi_{M}\right)$ is called a quasi-Poisson $G_{D}^{\mathfrak{h}}$-manifold.

Remark 3. A quasi-Poisson Lie group $G_{D}^{\mathfrak{h}}$ with the natural left action is not a quasi-Poisson $G_{D}^{\mathfrak{h}}$-manifold. In fact, $\left(\varphi_{\mathfrak{h}}\right)_{G}=\varphi_{\mathfrak{h}}^{R}$.

Finally we define a moment map for a quasi-Poisson action to carry out the deformation of symplectic structures using the moment map theory for quasi-Poisson actions in Section 3, We need some preliminaries to define a moment map. For any quasi-triple $(D, G, \mathfrak{h})$, since $G$ is a closed subgroup of $D$, the quotient space $D / G$ is a smooth manifold, which is the range of moment maps. The action of $D$ on itself by left multiplication induces an action of $D$ on $D / G$. We call it dressing action of $D$ on $D / G$ and denote the corresponding infinitesimal action by $X \mapsto X_{D / G}$ for $X$ in $\mathfrak{d}$. Let $p_{D / G}: D \rightarrow D / G$ be the natural projection. Then

$$
\pi_{D / G}^{\mathfrak{h}}:=p_{D / G *} \pi_{D}^{\mathfrak{h}}
$$

is a 2 -vector field on $D / G$. We consider the dressing action on $D / G$ restricted to $G$, and can see that $\pi_{D / G}^{\mathfrak{h}}$ satisfies (9) and (10). Therefore $\left(D / G, \pi_{D / G}^{\mathfrak{h}}\right)$ is a quasi-Poisson $G_{D}^{\mathfrak{h}}$-manifold. The following definition is one of the important notions to define moment maps.

Definition 5. An isotropic complement $\mathfrak{h}$ of $\mathfrak{g}$ in $\mathfrak{d}$ is called admissible at a point $s$ in $D / G$ if the infinitesimal dressing action restricted to $\mathfrak{h}$ defines an isomorphism from $\mathfrak{h}$ onto $T_{s}(D / G)$, that is, the map $\mathfrak{h} \rightarrow T_{s}(D / G), \xi \mapsto$ $\xi_{D / G, s}$ is an isomorphism. A quasi-triple $(D, G, \mathfrak{h})$ is complete if $\mathfrak{h}$ is admissible everywhere on $D / G$.

It is clear that any isotropic complement $\mathfrak{h}$ of $\mathfrak{g}$ is admissible at $e G$ in $D / G$. If the complement $\mathfrak{h}$ is admissible at a point $s$ in $D / G$, then it is also admissible on some open neighborhood $U$ of $s$. For a quasi-triple $(D, G, \mathfrak{h})$, we assume that $\mathfrak{h}$ is admissible on an open subset $U$ of $D / G$. Then for any $X$ in $\mathfrak{g}$, we define the 1-form $\hat{X}_{\mathfrak{h}}$ on $U$ by

$$
<\hat{X}_{\mathfrak{h}}, \xi_{D / G}>=(X \mid \xi)
$$


for any $\xi$ in $\mathfrak{h}$. If a quasi-triple $(D, G, \mathfrak{h})$ is complete, then $\hat{X}_{\mathfrak{h}}$ is a global 1 -form on $D / G$. Next we define a twist between isotropic complement subspaces $\mathfrak{h}$ and $\mathfrak{h}^{\prime}$ of $\mathfrak{g}$ in $\mathfrak{d}$. Twists also play an important role in the moment map theory for quasi-Poisson actions. Let $j$ and $j^{\prime}$ be the linear isomorphism (5) defined by Manin quasi-triples $(\mathfrak{d}, \mathfrak{g}, \mathfrak{h})$ and $\left(\mathfrak{d}, \mathfrak{g}, \mathfrak{h}^{\prime}\right)$ respectively. Consider the map

$$
t:=j^{\prime}-j: \mathfrak{g}^{*} \rightarrow \mathfrak{d} .
$$

It is easy to show that $t$ takes values in $\mathfrak{g}$ and that it is anti-symmetric, so that the map $t$ defines an element $t$ in $\Lambda^{2} \mathfrak{g}$ which we denote by the same letter. The element $t$ is called the twist from $\mathfrak{h}$ to $\mathfrak{h}^{\prime}$. Fix a quasi-triple $(D, G, \mathfrak{h})$. Let $\mathfrak{h}_{t}$ be an isotropic complement of $\mathfrak{g}$ with a twist $t$ from $\mathfrak{h}$. Then we can represent the elements $\varphi_{\mathfrak{h}_{t}}, F_{\mathfrak{h}_{t}}$ and $\pi_{G}^{\mathfrak{h}_{t}}$ defined by a quasitriple $\left(D, G, \mathfrak{h}_{t}\right)$ as follows:

$$
\begin{aligned}
\varphi_{\mathfrak{h}_{t}} & =\varphi_{\mathfrak{h}}+\frac{1}{2}[t, t]+\varphi_{t}, \\
F_{\mathfrak{h}_{t}} & =F_{\mathfrak{h}}+F_{t}, \\
\pi_{G}^{\mathfrak{h}_{t}} & =\pi_{G}^{\mathfrak{h}}+t^{L}-t^{R},
\end{aligned}
$$

where $[t, t]:=\left[t^{L}, t^{L}\right]_{e}, \varphi_{t}(\xi):=\overline{\operatorname{ad}_{\xi}^{*} t}$ and $F_{t}(X):=\operatorname{ad}_{X} t$. Here ad denotes the adjoint action of $\mathfrak{g}$ on $\Lambda^{2} \mathfrak{g}$ and $\overline{\operatorname{ad}_{\xi}^{*} t}$ denotes the projection of $\operatorname{ad}_{\xi}^{*} t$ onto $\Lambda^{2} \mathfrak{g} \subset \Lambda^{2} \mathfrak{d}$, where $\mathfrak{d}^{*}$ including $\mathfrak{g}^{*}$ acts on $\Lambda^{2} \mathfrak{d}$ by the coadjoint action. Let $\left\{e_{i}\right\}$ be a basis on $\mathfrak{g}$ and $\left\{\varepsilon^{i}\right\}$ be the basis on $\mathfrak{h}$ identified with the dual basis of $\left\{e_{i}\right\}$ on $\mathfrak{g}^{*}$ by $j^{-1}$. Then the basis $\left\{\varepsilon_{t}^{i}\right\}$ on $\mathfrak{h}_{t}$ identified with the dual basis of $\left\{e_{i}\right\}$ on $\mathfrak{g}^{*}$ by $j^{\prime-1}$ can be written by

$$
\varepsilon_{t}^{i}=\varepsilon^{i}+t^{i j} e_{j}
$$

where $t=\frac{1}{2} t^{i j} e_{i} \wedge e_{j}$. Moreover components of $\varphi_{t}$ with respect to the basis $\left\{\varepsilon^{i}\right\}$ are written as

$$
\varphi_{t}^{i j k}=\left(F_{\mathfrak{h}}\right)_{l}^{j k} t^{i l}-\left(F_{\mathfrak{h}}\right)_{l}^{i k} t^{j l}
$$

This indication is useful later. Let $\left(M, \pi_{M}^{\mathfrak{h}}\right)$ be a quasi-Poisson $G_{D}^{\mathfrak{h}}$-manifold. We set that $\pi_{M}^{\mathfrak{h}_{t}}:=\pi_{M}^{\mathfrak{h}}-t_{M}$. Then it follows that $\left(M, \pi_{M}^{\mathfrak{h}_{t}}\right)$ is a quasi-Poisson $G_{D}^{\mathfrak{h}_{t}}$-manifold. Now we define moment maps for quasi-Poisson actions.

Definition 6. Let $G_{D}^{\mathfrak{h}}$ be a connected quasi-Poisson Lie group defined by a quasi-triple $(D, G, \mathfrak{h})$ and $\left(M, \pi_{M}^{\mathfrak{h}}\right)$ be a quasi-Poisson $G_{D}^{\mathfrak{h}}$-manifold. Then a map $\mu: M \rightarrow D / G$ which is equivariant with respect to the $G$-action on 
$M$ and the dressing action on $D / G$ is a moment map for the quasi-Poisson action of $G_{D}^{\mathfrak{h}}$ on $\left(M, \pi_{M}^{\mathfrak{h}}\right)$ if for any open subset $\Omega \subset M$ and any isotropic complement $\mathfrak{h}^{\prime}$ admissible on $\mu(\Omega)$,

$$
\left(\pi_{M}^{\mathfrak{h}^{\prime}}\right)^{\sharp}\left(\mu^{*}\left(\hat{X}_{\mathfrak{h}^{\prime}}\right)\right)=X_{M}
$$

on $\Omega$ for any $X$ in $\mathfrak{g}$. Here $<\left(\pi_{M}^{\mathfrak{h}^{\prime}}\right)^{\sharp}(\alpha), \beta>:=\pi_{M}^{\mathfrak{h}^{\prime}}(\alpha, \beta)$. We call a quasiPoisson action with a moment map a quasi-Poisson-Hamiltonian action.

Actually we need not impose the equation (17) on all admissible complements because we have the following proposition.

Proposition 2.1 ([1]). Let $\mathfrak{h}$ and $\mathfrak{h}^{\prime}$ be two complements admissible at a point $s$ in $D / G$, and $p$ in $M$ be such that $\mu(p)=s$. Then, at the point $p$, conditions (17) for $\mathfrak{h}$ and $\mathfrak{h}^{\prime}$ are equivalent, namely

$$
\left(\pi_{M}^{\mathfrak{h}}\right)^{\sharp}\left(\mu^{*}\left(\hat{X}_{\mathfrak{h}}\right)\right)_{p}=\left(\pi_{M}^{\mathfrak{h}^{\prime}}\right)^{\sharp}\left(\mu^{*}\left(\hat{X}_{\mathfrak{h}^{\prime}}\right)\right)_{p} .
$$

For a quasi-Poisson manifold with a quasi-Poisson-Hamiltonian action, the following theorem holds.

Theorem $2.2([1])$. Let $\left(M, \pi_{M}^{\mathfrak{h}}\right)$ be a quasi-Poisson manifold on which a quasi-Poisson Lie group $G_{D}^{\mathfrak{h}}$ defined by a quasi-triple $(D, G, \mathfrak{h})$ acts by a quasi-Poisson-Hamiltonian action $\sigma$. For any $p$ in $M$, if both $\mathfrak{h}^{\prime}$ and $\mathfrak{h}^{\prime \prime}$ are admissible at $\mu(p)$ in $D / G$, then

$$
\operatorname{Im}\left(\pi_{M}^{\mathfrak{h}^{\prime}}\right)_{p}^{\sharp}=\operatorname{Im}\left(\pi_{M}^{\mathfrak{h}^{\prime \prime}}\right)_{p}^{\sharp},
$$

where $\mu$ is a moment map for $\sigma$.

Now we show important examples for quasi-Poisson-Hamiltonian actions.

Example 1 (Poisson manifolds [1, [2], [6]). Let $(M, \pi)$ be a Poisson manifold on which a connected Poisson Lie group $\left(G, \pi_{G}\right)$ acts by a Poisson action $\sigma$. Then $(M, \pi)$ is a quasi-Poisson $\left(G, \pi_{G}, 0\right)$-manifold and $\sigma$ is a quasi-Poisson action on $(M, \pi)$. In fact, the Manin triple $\left(\mathfrak{g} \oplus \mathfrak{g}^{*}, \mathfrak{g}, \mathfrak{g}^{*}\right)$ corresponding to $\left(G, \pi_{G}\right)$ is a Manin quasi-triple and the multiplicative 2 -vector field $\pi_{G}$ on $G$ coincides with the 2-vector field $\pi_{G}^{\mathfrak{g}^{*}}$ defined by the corresponding quasi-triple $\left(D, G, \mathfrak{g}^{*}\right)$. Since $[\pi, \pi]=0$ and the Poisson action $\sigma$ satisfies

$$
\mathfrak{L}_{X_{M}} \pi=F_{\mathfrak{g}^{*}}(X)_{M}
$$

for any $X$ in $\mathfrak{g}$, the action $\sigma$ is a quasi-Poisson action by Definition 4. Here the dual of $F_{\mathfrak{g}^{*}}$ coincides with the bracket on $\mathfrak{g}^{*}$ defined by $\left(G, \pi_{G}\right)$. 
We assume that $\pi_{G}$ is complete and that there exists a G-equivariant moment map $\mu: M \rightarrow G^{*}$ for the Poisson action $\sigma$, where $G^{*}$ is the dual Poisson Lie group of $\left(G, \pi_{G}\right)$ and $G$ acts on $G^{*}$ by the dressing action in the sense of Lu and Weinstein [6]. Then $\sigma$ is a quasi-Poisson-Hamiltonian action. Actually, by the definition, the map $\mu$ satisfies

$$
\pi^{\sharp}\left(\mu^{*}\left(X^{L}\right)\right)=X_{M}
$$

for any $X$ in $\mathfrak{g}$, where $X^{L}$ is a left-invariant 1 -form on $G^{*}$ with value $X$ at $e$ in $G^{*}$. The quotient manifold $D / G$ is diffeomorphic to $G^{*}$ as a manifold. The quasi-triple $\left(D, G, \mathfrak{g}^{*}\right)$ is complete since $\pi_{G}$ is complete. Then 1-form $\hat{X}_{\mathfrak{g}^{*}}$ defined by (11) is global for any $X$ in $\mathfrak{g}$. Furthermore the 1-form $\hat{X}_{\mathfrak{g}^{*}}$ on $D / G \cong G^{*}$ coincides with $X^{L}$. The complement $\mathfrak{g}^{*}$ is admissible at any point in $D / G$, so that the map $\mu: M \rightarrow G^{*} \cong D / G$ is a moment map for the quasi-Poisson action $\sigma$ because of (19) and Proposition 2.1.

Example 2 (symplectic manifolds [1], [9]). Let $(M, \omega)$ be a symplectic manifold on which a connected Lie group $G$ acts by a symplectic-Hamiltonian action $\sigma$. Since the symplectic structure $\omega$ induces a Poisson structure $\pi$, the pair $(M, \pi)$ is a Poisson manifold. Then the action $\sigma$ is a Poisson action of a trivial Poisson Lie group $(G, 0)$ on $(M, \pi)$. The trivial Poisson structure 0 on $G$ is complete and the quasi-triple corresponding to $(G, 0)$ is $\left(T^{*} G, G, \mathfrak{g}^{*}\right)$, where $T^{*} G \cong G \times \mathfrak{g}^{*}$ is the cotangent bundle of $G$ equipped with the group structure of a semi-direct product with respect to coadjoint action of $G$ on $\mathfrak{g}^{*}$ (See [1]). The dual group $G^{*}$ of $(G, 0)$ is the additive group $\mathfrak{g}^{*}$ and the moment map $\mu$ for symplectic action $\sigma$ is $G$-equivariant with respect to $\sigma$ on $M$ and $A d^{*}$ on $\mathfrak{g}^{*}$ by the definition. Furthermore the dressing action of $G$ on $G^{*}=\mathfrak{g}^{*}$ coincides with the coadjoint action $\mathrm{Ad}^{*}$. Thus the map $\mu: M \rightarrow \mathfrak{g}^{*}=G^{*}$ is a moment map for the Poisson action $\sigma$. Therefore, by Example 1, the map $\mu: M \rightarrow \mathfrak{g}^{*} \cong T^{*} G / G$ is a moment map for the quasi-Poisson action $\sigma$ on the quasi-Poisson $(G, 0,0)$-manifold $(M, \pi)$.

\section{Main Result}

In this section, we carry out deformations of symplectic structures on a smooth manifold. We use the moment map theory for quasi-Poisson actions for it. A moment map for the quasi-Poisson action on a quasi-Poisson $G_{D^{\mathfrak{h}}}^{\mathfrak{h}}$-manifold $\left(M, \pi_{M}^{\mathfrak{h}}\right)$ are defined with the conditions for the family of quasiPoisson $G_{D^{-}}^{\mathfrak{h}^{\prime}}$-structures $\left\{\pi_{M}^{\mathfrak{h}^{\prime}}\right\}_{\mathfrak{h}^{\prime}}$ on $M$. For each complement $\mathfrak{h}^{\prime}$, there exists 
a twist $t$ in $\Lambda^{2} \mathfrak{g}$ such that $\mathfrak{h}^{\prime}=\mathfrak{h}_{t}$, so that the family $\left\{\pi_{M}^{\mathfrak{h}^{\prime}}\right\}_{\mathfrak{h}^{\prime}}$ is regarded as the family parametrized by twist, $\left\{\pi_{M}^{\mathfrak{h}_{t}}\right\}_{t \in \Lambda^{2} \mathfrak{g}}$. When the quasi-Poisson $G_{D}^{\mathfrak{h}_{t}}$-structure with twist $t=0$ is induced by a given symplectic structure, we will give the method of finding a quasi-Poisson $G_{D}^{\mathfrak{h} t}$-structure which induced a symplectic structure in $\left\{\pi_{M}^{\mathfrak{h}_{t}}\right\}_{t}$. That is, we can deform a given symplectic structure to a new one by a twist $t$. This deformation can be carried out due to using the family $\left\{\pi_{M}^{\mathfrak{h}_{t}}\right\}_{t}$ as moment map conditions for quasi-Poisson actions. In this regard, it is described as follows in [1]: It would be interesting to find a geometric framework for considering the family $\left\{\pi_{M}^{\mathfrak{h}_{t}}\right\}_{t}$. Our deformation is one of the answers for this proposal.

Let $(M, \omega)$ be a symplectic manifold on which an $n$-dimensional connected Lie group $G$ acts by symplectic-Hamiltonian action $\sigma$ with a moment map $\mu: M \rightarrow \mathfrak{g}^{*}$. Let $\pi$ be the nondegenerate Poisson structure on $M$ induced by $\omega$. Then $\mu$ is a moment map for the quasi-Poisson-Hamiltonian action $\sigma$ of $(G, 0,0)$ on $(M, \pi)$ by Example 2 in Section 2 .

Let $\left(\mathfrak{g} \oplus \mathfrak{g}^{*}, \mathfrak{g}, \mathfrak{g}^{*}\right)$ be the Manin triple corresponding to the trivial Poisson Lie group $(G, 0)$, where $\mathfrak{g} \oplus \mathfrak{g}^{*}$ has the Lie bracket

$$
[X, Y]=[X, Y]_{\mathfrak{g}}, \quad[X, \xi]=\operatorname{ad}_{X}^{*} \xi, \quad[\xi, \eta]=[\xi, \eta]_{\mathfrak{g}^{*}}=0
$$

for any $X, Y$ in $\mathfrak{g}$ and $\xi, \eta$ in $\mathfrak{g}^{*}$. Here the bracket $[\cdot, \cdot]_{\mathfrak{g}}$ and $[\cdot, \cdot]_{\mathfrak{g}^{*}}$ are the brackets on $\mathfrak{g}$ and $\mathfrak{g}^{*}$ respectively. Then the Manin (quasi-)triple $(\mathfrak{g} \oplus$ $\mathfrak{g}^{*}, \mathfrak{g}, \mathfrak{g}^{*}$ ) defines $F:=F_{\mathfrak{g}^{*}}=0$ and $\varphi:=\varphi_{\mathfrak{g}^{*}}=0$ (see (6) and (7)). Since the corresponding quasi-triple $\left(T^{*} G, G, \mathfrak{g}^{*}\right)$ is complete by Example 1 and 2. an isotropic complement $\mathfrak{g}^{*}$ is admissible at any $\xi$ in $\mathfrak{g}^{*}$ by Definition 5 , and hence it is admissible at any $\xi$ in $\mu(M)$.

Let $\mathfrak{g}_{t}^{*}$ be an isotropic complement of $\mathfrak{g}$ in $\mathfrak{g} \oplus \mathfrak{g}^{*}$ with a twist $t$ in $\Lambda^{2} \mathfrak{g}$ from $\mathfrak{g}^{*}$. When we deform $\pi$ to $\pi_{M}^{t}:=\pi-t_{M}$ by a twist $t$, the quasi-Poisson Lie group $(G, 0,0)$ is deformed to $\left(G, \pi_{G}^{t}, \varphi_{\mathfrak{g}_{t}^{*}}\right)$, where $\pi_{G}^{t}=t^{L}-t^{R}$ and $\varphi_{\mathfrak{g}_{t}^{*}}=\frac{1}{2}[t, t]+\varphi_{t}$ by (12) and (14). Moreover it follows from $F=0$ and (16) that $\varphi_{t}=0$. So $\varphi_{\mathfrak{g}_{t}^{*}}=\frac{1}{2}[t, t]$.

On the other hand, it follows from Definition 4 that the quasi-Poisson $\left(G, \pi_{G}^{t}, \varphi_{\mathfrak{g}_{t}^{*}}\right)$-manifold $\left(M, \pi_{M}^{t}\right)$ satisfies

$$
\begin{aligned}
\frac{1}{2}\left[\pi_{M}^{t}, \pi_{M}^{t}\right] & =\left(\varphi_{\mathfrak{g}_{t}^{*}}\right)_{M}, \\
\mathfrak{L}_{X_{M}} \pi_{M}^{t} & =F_{\mathfrak{g}_{t}^{*}}(X)_{M} .
\end{aligned}
$$


If $\left(\varphi_{\mathfrak{g}_{t}^{*}}\right)_{M}=0$, i.e., $[t, t]_{M}=0$, then the 2-vector field $\pi_{M}^{t}$ is a Poisson structure on $M$ by (21).

Assume that a twist $t$ in $\Lambda^{2} \mathfrak{g}$ is an r-matrix, namely that $[t, t]$ is adinvariant. Then $\pi_{G}^{t}=t^{L}-t^{R}$ is a multiplicative Poisson structure (see [6]). Therefore $\left(G, \pi_{G}^{t}\right)$ is a Poisson Lie group. Then it follows that $F_{\mathfrak{g}_{t}^{*}}$ coincides with the dual of the bracket map $[\cdot, \cdot]_{\pi_{G}^{t}}: \mathfrak{g}^{*} \wedge \mathfrak{g}^{*} \rightarrow \mathfrak{g}^{*}$ on $\mathfrak{g}^{*}$ defined by the Poisson Lie group $\left(G, \pi_{G}^{t}\right)$. In fact, by the relation (19), we have

$$
F_{\mathfrak{g}_{t}^{*}}^{*}(\xi, \eta)=\operatorname{ad}_{t^{\sharp}(\xi)} \eta-\operatorname{ad}_{t^{\sharp}(\eta)} \xi,
$$

where $\left\langle t^{\sharp}(\xi), \eta>:=t(\xi, \eta)\right.$. And the bracket on $\mathfrak{g}^{*}$ induced by a multiplicative Poisson structure defined by an r-matrix is represented by the right-hand side of (23) (see [5], Ex.2.19). Therefore, since $G$ is connected, the condition (22) means that the action $\sigma$ is a Poisson action of $\left(G, \pi_{G}^{t}\right)$ on $\left(M, \pi_{M}^{t}\right)$ under the assumption that $t$ is an r-matrix and that $[t, t]_{M}=0$.

Let $\left\{e_{i}\right\}$ be a basis on $\mathfrak{g}$, a set $\left\{\varepsilon^{i}\right\}$ be the dual basis of $\left\{e_{i}\right\}$ on $\mathfrak{g}^{*}$. Then we can write by (15),

$$
\mathfrak{g}_{t}^{*}=\operatorname{span}\left\{\varepsilon^{i}+t^{i j} e_{j} \mid i=1, \ldots, n\right\},
$$

where $t=\frac{1}{2} t^{i j} e_{i} \wedge e_{j}$ in $\Lambda^{2} \mathfrak{g}$. If $\mathfrak{g}_{t}^{*}$ is admissible at any point in $\mu(M)$, then it satisfies $\operatorname{Im} \pi_{p}^{\sharp}=\operatorname{Im}\left(\pi_{M}^{t}\right)_{p}^{\sharp}$ for any $p$ in $M$ by Theorem 2.2. The nondegeneracy of $\pi$ means that $\operatorname{Im} \pi_{p}^{\sharp}=T_{p} M$ for any $p$ in $M$. Therefore, by the fact that $\operatorname{Im}\left(\pi_{M}^{t}\right)_{p}^{\sharp}=T_{p} M$ for any $p$ in $M$, a quasi-Poisson structure $\pi_{M}^{t}$ is also nondegenerate.

Here we shall examine the condition for a isotropic complement to be admissible at a point in $\mathfrak{g}^{*}$ in more detail. Let $\left(\xi_{i}\right)$ be the linear coordinates for $\left\{\varepsilon^{i}\right\}$. Then it follows that for $i=1, \ldots, n$,

$$
\begin{aligned}
\left(\varepsilon^{i}+t^{i j} e_{j}\right)_{\mathfrak{g}^{*}} & =-\frac{\partial}{\partial \xi_{i}}+t^{i j} c_{j l}^{k} \xi_{k} \frac{\partial}{\partial \xi_{l}} \\
& =-t^{i j} \sum_{l \neq i} c_{l j}^{k} \xi_{k} \frac{\partial}{\partial \xi_{l}}-\left(1+t^{i j} c_{i j}^{k} \xi_{k}\right) \frac{\partial}{\partial \xi_{i}},
\end{aligned}
$$

where $X \mapsto X_{\mathfrak{g}^{*}}$, for $X$ in $\mathfrak{g} \oplus \mathfrak{g}^{*}$, is the infinitesimal action of the dressing action on $\mathfrak{g}^{*} \cong T^{*} G / G$. The quasi-triple $\left(T^{*} G, G, \mathfrak{g}_{t}^{*}\right)$ is complete if and only if the elements (25) form a basis on $T_{\xi}\left(\mathfrak{g}^{*}\right) \cong \mathfrak{g}^{*}$ for any $\xi=\left(\xi_{1}, \ldots, \xi_{n}\right)$. Hence this means that the matrix

$$
A_{t}(\xi):=\left(\begin{array}{cccc}
-1-t^{1 j} c_{1 j}^{k} \xi_{k} & -t^{1 j} c_{2 j}^{k} \xi_{k} & \cdots & -t^{1 j} c_{n j}^{k} \xi_{k} \\
-t^{2 j} c_{1 j}^{k} \xi_{k} & -1-t^{2 j} c_{2 j}^{k} \xi_{k} & \cdots & -t^{2 j} c_{n j}^{k} \xi_{k} \\
\vdots & \vdots & \ddots & \vdots \\
-t^{n j} c_{1 j}^{k} \xi_{k} & -t^{n j} c_{2 j}^{k} \xi_{k} & \cdots & -1-t^{n j} c_{n j}^{k} \xi_{k}
\end{array}\right)
$$


is regular for any $\xi$. Therefore this is equivalent to $f_{t}\left(\xi_{1}, \ldots, \xi_{n}\right):=\operatorname{det} A_{t}(\xi) \neq$ 0 . Since the constant term of $f_{t}$ is $(-1)^{n}$ and since coefficients of the rest of the term include $t^{i j}$ 's, a family of hypersurfaces $\left\{f_{t}=0\right\}_{t \in \Lambda^{2} \mathfrak{g}}$ in $\mathfrak{g}^{*}$ diverges to infinity as $t$ approaches the origin 0 in $\Lambda^{2} \mathfrak{g}$. If $M$ is compact, then $\mu(M)$ is bounded. So it follows that an intersection of $\left\{f_{t}=0\right\}$ and $\mu(M)$ is empty for a twist $t$ close sufficiently to the origin. Therefore since $\mathfrak{g}_{t}^{*}$ is admissible on $\mu(M)$, the 2 -vector field $\pi_{M}^{t}$ is nondegenerate.

Since any nondegenerate Poisson structure on $M$ defines a symplectic structure on $M$, the following theorem holds.

Theorem 3.1. Let $(M, \omega)$ be a symplectic manifold on which a connected Lie group $G$ with the Lie algebra $\mathfrak{g}^{*}$ acts by a symplectic-Hamiltonian action $\sigma$ and $\mu$ be a moment map for $\sigma$. Then the following holds:

1. If a twist $t$ in $\Lambda^{2} \mathfrak{g}$ satisfies that $[t, t]_{M}=0$, then $t$ deforms the Poisson structure $\pi$ induced by $\omega$ to a Poisson structure $\pi_{M}^{t}:=\pi-t_{M}$. Moreover, if $t$ is an r-matrix, then $\sigma$ is a Poisson action of $\left(G, \pi_{G}^{t}\right)$ on $\left(M, \pi_{M}^{t}\right)$, where $\pi_{G}^{t}=t^{L}-t^{R}$.

2. For a twist $t$ in $\Lambda^{2} \mathfrak{g}$, if the isotropic complement $\mathfrak{g}_{t}^{*}$ is admissible on $\mu(M)$, then $t$ deforms the nondegenerate 2-vector field $\pi$ induced by $\omega$ to a nondegenerate 2-vector field $\pi_{M}^{t}$. In particular, if $M$ is compact, then a 2-vector field $\pi_{M}^{t}$ is nondegenerate for a twist $t$ close sufficiently to the origin 0 in $\Lambda^{2} \mathfrak{g}$.

Therefore, if a twist $t$ satisfies the assumptions of both 1 and [2, then $t$ deforms $\omega$ to a symplectic structure $\omega^{t}$ induced by the nondegenerate Poisson structure $\pi_{M}^{t}$.

The following theorem gives a sufficient condition for a twist to deform a symplectic structure.

Theorem 3.2. Let $(M, \omega)$ be a symplectic manifold on which an $n$-dimensional connected Lie group $G$ acts by a symplectic-Hamiltonian action $\sigma$. Assume that $X, Y$ in $\mathfrak{g}$ satisfy $[X, Y]=0$. Then the twist $t=\frac{1}{2} X \wedge Y$ in $\Lambda^{2} \mathfrak{g}$ deforms the symplectic structure $\omega$ to a symplectic structure $\omega_{t}$. For example, a twist $t$ in $\Lambda^{2} \mathfrak{h}$, where $\mathfrak{h}$ is a Cartan subalgebra of $\mathfrak{g}$, satisfies the assumption of the theorem.

Proof. For $X$ and $Y$ in $\mathfrak{g}$, we set

$$
X=X^{i} e_{i}, Y=Y^{j} e_{j},
$$


where $\left\{e_{i}\right\}_{i=1}^{n}$ is a basis on the Lie algebra $\mathfrak{g}$. Then since $[X, Y]=X^{i} Y^{j} c_{i j}^{k} e_{k}=$ 0 , we obtain the following conditions:

$$
X^{i} Y^{j} c_{i j}^{k}=0 \text { for any } k,
$$

where $c_{i j}^{k}$ are the structure constants of $\mathfrak{g}$ with respect to the basis $\left\{e_{i}\right\}$. Moreover, since we have

$$
[t, t]=\left[\frac{1}{2} X \wedge Y, \frac{1}{2} X \wedge Y\right]=\frac{1}{2} X \wedge[X, Y] \wedge Y=0
$$

the twist $t$ is an r-matrix such that $[t, t]_{M}=0$ obviously. Hence $\pi_{M}^{t}$ is a Poisson structure, and if $\pi_{M}^{t}$ is nondegenerate, then twist $t$ induces the symplectic structure $\omega_{t}$.

We shall show the nondegeneracy of $\pi_{M}^{t}$. Let $\mu$ be the moment map for a given symplectic-Hamiltonian action $\psi$. Then the nondegeneracy of $\pi_{M}^{t}$ means that $\mathfrak{g}_{t}^{*}$ is admissible at any point in $\mu(M)$. We prove a stronger condition that the quasi-triple $\left(T^{*} G, G, \mathfrak{g}_{t}^{*}\right)$ is complete.

Let $\left\{\varepsilon^{i}\right\}$ be the dual basis of $\left\{e_{i}\right\}$ on $\mathfrak{g}^{*}$ and $\left(\xi_{i}\right)$ be the linear coordinates for $\left\{\varepsilon^{i}\right\}$. Since $t=\frac{1}{2} X^{i} Y^{j} e_{i} \wedge e_{j}$,

$$
\mathfrak{g}_{t}^{*}=\operatorname{span}\left\{\varepsilon^{i}+X^{i} Y^{j} e_{j} \mid i=1, \cdots n\right\} .
$$

Then it follows that for $i=1, \ldots, n$,

$$
\left(\varepsilon^{i}+X^{i} Y^{j} e_{j}\right)_{\mathfrak{g}^{*}}=-X^{i} Y^{j} \sum_{l \neq i} c_{l j}^{k} \xi_{k} \frac{\partial}{\partial \xi_{l}}-\left(1+X^{i} Y^{j} c_{i j}^{k} \xi_{k}\right) \frac{\partial}{\partial \xi_{i}}
$$

The quasi-triple $\left(T^{*} G, G, \mathfrak{g}_{t}^{*}\right)$ is complete if and only if the elements (27) form a basis on $T_{\xi}\left(\mathfrak{g}^{*}\right) \cong \mathfrak{g}^{*}$ for any $\xi=\left(\xi_{1}, \ldots, \xi_{n}\right)$. Therefore we shall prove that the matrix

$$
\left(\begin{array}{cccc}
-1-X^{1} Y^{j} c_{1 j}^{k} \xi_{k} & -X^{1} Y^{j} c_{2 j}^{k} \xi_{k} & \cdots & -X^{1} Y^{j} c_{n j}^{k} \xi_{k} \\
-X^{2} Y^{j} c_{1 j}^{k} \xi_{k} & -1-X^{2} Y^{j} c_{2 j}^{k} \xi_{k} & \cdots & -X^{2} Y^{j} c_{n j}^{k} \xi_{k} \\
\vdots & \vdots & \ddots & \vdots \\
-X^{n} Y^{j} c_{1 j}^{k} \xi_{k} & -X^{n} Y^{j} c_{2 j}^{k} \xi_{k} & \cdots & -1-X^{n} Y^{j} c_{n j}^{k} \xi_{k}
\end{array}\right)
$$

is regular. In the case of $X=0$, this matrix is equal to the opposite of the identity matrix, so that it is regular. In the case of $X \neq 0$, using $X^{i} Y^{j} c_{i j}^{k}=0$, we can transform the matrix to the opposite of the identity matrix. Thus the matrix (28) is regular. Therefore $\mathfrak{g}_{t}^{*}$ is admissible at any point in $\mathfrak{g}^{*}$. That is, $\left(T^{*} G, G, \mathfrak{g}_{t}^{*}\right)$ is complete. 
Remark 4. We try to generalize the assumption of Theorem 3.2 and consider $X, Y$ in $\mathfrak{g}$ such that $[X, Y]=a X+b Y(a, b$ in $\mathbb{R})$, that is, the subspace spanned by $X, Y$ is also a Lie subalgebra. We set $t=\frac{1}{2} X \wedge Y$ in $\Lambda^{2} \mathfrak{g}$. Since

$$
[t, t]=\frac{1}{2} X \wedge[X, Y] \wedge Y=\frac{1}{2} X \wedge(a X+b Y) \wedge Y=0
$$

the twist $t$ is an r-matrix such that $[t, t]_{M}=0$. Therefore the symplectic action $\psi$ is a Poisson action of $\left(G, \pi_{G}^{t}\right)$ on $\left(M, \pi_{M}^{t}\right)$. Then we research whether $\mathfrak{g}_{t}^{*}$ is admissible at all points in $\mathfrak{g}^{*}$. Similarly to the proof of Theorem 3.2, a matrix to check the regularity can be deformed to

$$
\left(\begin{array}{cccc}
-1-\left(a X^{k}+b Y^{k}\right) \xi_{k} & 0 & \cdots & 0 \\
0 & -1 & \cdots & 0 \\
\vdots & \vdots & \ddots & \vdots \\
0 & 0 & \cdots & -1
\end{array}\right)
$$

Therefore this matrix is regular if and only if

$$
-1-\left(a X^{k}+b Y^{k}\right) \xi_{k} \neq 0 .
$$

In the case of $[X, Y]=0$, by Theorem 3.2, the space $\mathfrak{g}_{t}^{*}$ is admissible at all points in $\mathfrak{g}^{*}$. In the case of $[X, Y] \neq 0$, we shall denote by $\cdot$ the standard inner product on $\mathfrak{g}^{*} \cong \mathbb{R}^{n}$. Then the above condition means

$$
\eta_{[X, Y]} \cdot \xi \neq-1
$$

where $\eta_{X}=\sum_{k} X^{k} \varepsilon^{k}$ for $X=X^{k} e_{k}$ in $\mathfrak{g}$. Let $\xi^{\prime}$ be an element which is not orthogonal to $\eta_{[X, Y]}$. By setting

$$
\xi:=-\frac{\xi^{\prime}}{\eta_{[X, Y]} \cdot \xi^{\prime}},
$$

we obtain $\eta_{[X, Y]} \cdot \xi=-1$, so that $\mathfrak{g}_{t}^{*}$ is not admissible at $\xi$. Eventually, to make sure of the admissibility of $\mathfrak{g}_{t}^{*}$, we need check whether such a point $\xi$ is included in $\mu(M)$.

\section{Deformations of the canonical symplectic form on $\mathbb{C P}^{n}$ and $\operatorname{Gr}\left(r ; \mathbb{C}^{n}\right)$}

In this section, we compute specifically which element $t$ in $\Lambda^{2} \mathfrak{g}$ defines a different symplectic structure $\omega_{t}$ from given one $\omega$ on a smooth manifold. One 
example is the complex projective space $\left(\mathbb{C P}^{k}, \omega_{\mathrm{FS}}\right)$, where $\omega_{\mathrm{FS}}$ is the FubiniStudy form, with an action of $\mathrm{SU}(k+1), k=1,2$. Another is $\left(\mathbb{C P}^{n}, \omega_{\mathrm{FS}}\right)$ with an action of the torus group $\mathbb{T}^{n}$. The other is the complex Grassmannian $\left(\operatorname{Gr}\left(r ; \mathbb{C}^{n}\right), \omega_{\mathrm{KK}}\right)$, where $\omega_{\mathrm{KK}}$ is the Kirillov-Kostant form, with an action of $\mathrm{SU}(k+1)$.

First we review the relation between $\mathrm{SU}(n+1)$ and $\mathbb{C P}^{n}$. For any $\left[z_{1}: \cdots: z_{n+1}\right]$ in $\mathbb{C P}^{n}$ and $g=\left(a_{i j}\right)$ in $\mathrm{SU}(n+1)$, the action is given by

$$
g \cdot\left[z_{1}: \cdots: z_{n+1}\right]:=\left[\sum_{j=1}^{n+1} a_{1 j} z_{j}: \cdots: \sum_{j=1}^{n+1} a_{n+1, j} z_{j}\right] .
$$

The isotropic subgroup of $[1: 0: \cdots: 0]$ is

$$
\mathrm{S}(\mathrm{U}(1) \times \mathrm{U}(n))=\left\{\left(\begin{array}{cc}
e^{i \theta} & O \\
O & B
\end{array}\right) \in \mathrm{SU}(n+1) \mid \theta \in \mathbb{R}, B \in U(n)\right\} .
$$

Therefore it follows

$$
\mathrm{SU}(n+1) / \mathrm{S}(\mathrm{U}(1) \times \mathrm{U}(n)) \cong \mathbb{C P}^{n} .
$$

The complex projective space $\mathbb{C P}^{n}$ has the coordinate neighborhood system $\left\{\left(U_{i}, \varphi_{i}\right)\right\}_{i}$ consisting of $n+1$ open sets $U_{i}$ given by

$$
\begin{aligned}
& U_{i}:=\left\{\left[z_{1}: \cdots: z_{n+1}\right] \in \mathbb{C P}^{n} \mid z_{i} \neq 0\right\} \\
& \varphi_{i}: U_{i} \rightarrow \mathbb{C}^{n} \cong \mathbb{R}^{2 n} \\
& {\left[z_{1}: \cdots: z_{n+1}\right] } \mapsto\left(\frac{z_{1}}{z_{i}}, \ldots, \frac{z_{i-1}}{z_{i}}, \frac{z_{i+1}}{z_{i}}, \ldots, \frac{z_{n+1}}{z_{i}}\right) \\
& \mapsto\left(\operatorname{Re} \frac{z_{1}}{z_{i}}, \operatorname{Im} \frac{z_{1}}{z_{i}}, \ldots, \operatorname{Re} \frac{z_{n+1}}{z_{i}}, \operatorname{Im} \frac{z_{n+1}}{z_{i}}\right),
\end{aligned}
$$

for $i=1, \ldots, n+1$. By using this coordinate system, the Fubini-Study form $\omega_{\mathrm{FS}}$ on $\mathbb{C P}^{n}$ is defined by setting

$$
\varphi_{i}^{*}\left(\frac{i}{2} \partial \bar{\partial} \log \left(\sum_{j}\left|z_{j}\right|^{2}+1\right)\right)
$$

on each $U_{i}$.

The action of $\mathrm{SU}(n+1)$ on $\left(\mathbb{C P}^{n}, \omega_{\mathrm{FS}}\right)$ is a symplectic-Hamiltonian action and its moment map $\mu$ satisfies

$$
<\mu\left(\left[z_{1}: \cdots: z_{n+1}\right]\right), X>=\frac{1}{2} \operatorname{Im} \frac{\left\langle{ }^{t}\left(z_{1}, \ldots, z_{n+1}\right), X^{t}\left(z_{1}, \ldots, z_{n+1}\right)\right\rangle}{\left\langle{ }^{t}\left(z_{1}, \ldots, z_{n+1}\right),{ }^{t}\left(z_{1}, \ldots, z_{n+1}\right)\right\rangle}
$$


for any $\left[z_{1}: \cdots: z_{n+1}\right]$ in $\mathbb{C P}^{n}$ and $X$ in $\mathfrak{s u}(n+1)$.

We use

$X_{i j}$ : the $(i, j)$-element is 1 , the $(j, i)$-element is -1 , and the rest are 0 ,

$Y_{i j}:$ the $(i, j)$ - and $(j, i)$-elements are $i$, and the rest are 0 ,

$Z_{k} \quad$ : the $(k, k)$-element is $i$, and the $(n+1, n+1)$-element is $-i$

for $1 \leq i<j \leq n+1$ and $k=1, \ldots, n$, as a basis of $\mathfrak{s u}(n+1)$ which is defined by a Chevalley basis of the complexified Lie algebra $\mathfrak{s l}(n+1, \mathbb{C})$ of $\mathfrak{s u}(n+1)$. The subspace spanned by $Z_{k}$ 's is a Cartan subalgebra of $\mathfrak{s u}(n+1)$.

We consider the case of $n=1$. The complex projective line $\mathbb{C P}^{1}$ has the coordinate neighborhood system $\left\{\left(U_{1}, \varphi_{1}\right),\left(U_{2}, \varphi_{2}\right)\right\}$ given by

$$
\begin{aligned}
& U_{i}:=\left\{\left[z_{1}: z_{2}\right] \in \mathbb{C P}^{1} \mid z_{i} \neq 0\right\}(i=1,2), \\
& \varphi_{1}: U_{1} \rightarrow \mathbb{C} \cong \mathbb{R}^{2},\left[z_{1}: z_{2}\right] \mapsto \frac{z_{2}}{z_{1}} \mapsto\left(\operatorname{Re} \frac{z_{2}}{z_{1}}, \operatorname{Im} \frac{z_{2}}{z_{1}}\right), \\
& \varphi_{2}: U_{2} \rightarrow \mathbb{C} \cong \mathbb{R}^{2},\left[z_{1}: z_{2}\right] \mapsto \frac{z_{1}}{z_{2}} \mapsto\left(\operatorname{Re} \frac{z_{1}}{z_{2}}, \operatorname{Im} \frac{z_{1}}{z_{2}}\right) .
\end{aligned}
$$

The Fubini-Study form $\omega_{\mathrm{FS}}$ on $\mathbb{C P}^{1}$ is

$$
\omega_{\mathrm{FS}}=\frac{d x_{1} \wedge d y_{1}}{\left(x_{1}^{2}+y_{1}^{2}+1\right)^{2}}
$$

on $U_{1}$, where $\left(x_{1}, y_{1}\right):=\left(\operatorname{Re} \frac{z_{2}}{z_{1}}, \operatorname{Im} \frac{z_{2}}{z_{1}}\right)$. Then a moment map $\mu: \mathbb{C P}^{1} \rightarrow$ $\mathfrak{s u}(2)^{*}$ for the natural action of $\mathrm{SU}(2)$ on $\left(\mathbb{C P}^{1}, \omega_{\mathrm{FS}}\right)$ is defined by

$$
<\mu\left(\left[z_{1}: z_{2}\right]\right), X>=-\frac{1}{2} \operatorname{Im} \frac{\left\langle{ }^{t}\left(z_{1}, z_{2}\right), X^{t}\left(z_{1}, z_{2}\right)\right\rangle}{\left\langle{ }^{t}\left(z_{1}, z_{2}\right),{ }^{t}\left(z_{1}, z_{2}\right)\right\rangle}
$$

for any $\left[z_{1}: z_{2}\right]$ in $\mathbb{C P}^{1}$ and $X$ in $\mathfrak{s u}(2)$. Then $e_{1}:=X_{12}, e_{2}:=Y_{12}$ and $e_{3}:=Z_{1}$ form a basis of $\mathfrak{s u}(2)$. Let $\left\{\varepsilon^{i}\right\}$ be the dual basis of $\mathfrak{s u}(2)^{*}$. We obtain

$$
\mu\left(x_{1}, y_{1}\right)=\frac{y_{1}}{1+x_{1}^{2}+y_{1}^{2}} \varepsilon^{1}+\frac{x_{1}}{1+x_{1}^{2}+y_{1}^{2}} \varepsilon^{2}+\frac{1-x_{1}^{2}-y_{1}^{2}}{2\left(1+x_{1}^{2}+y_{1}^{2}\right)} \varepsilon^{3} .
$$

Hence $\mu\left(\mathbb{C P}^{1}\right) \subset \mathfrak{s u}(2)^{*}$ is the 2 -sphere with center at the origin and with radius $\frac{1}{2}$.

Let $\left(\xi_{i}\right)$ be the linear coordinates for $\left\{\varepsilon^{i}\right\}$. We set $\mathfrak{g}:=\mathfrak{s u}(2)$. Any twist $t$ is an r-matrix on $\mathfrak{g}$ because $e_{1} \wedge e_{2} \wedge e_{3}$ is ad-invariant. Since $\mathbb{C P}^{1}$ 
is 2-dimensional, it follows that $[t, t]_{\mathbb{C P}^{1}}=0$. Therefore we can deform the Poisson structure $\pi_{\mathrm{FS}}$ induced by $\omega_{\mathrm{FS}}$ to a Poisson structure $\pi_{\mathrm{FS}}^{t}$ on $\mathbb{C P}^{1}$ by $t$ and the natural action is a Poisson action of $\left(\mathrm{SU}(2), t^{L}-t^{R}\right)$.

Let $\mathfrak{g}_{t}^{*}$ be the space twisted $\mathfrak{g}^{*}$ by $t$ in $\Lambda^{2} \mathfrak{g}$. We consider what is the condition for $t$ under which $\mathfrak{g}_{t}^{*}$ is admissible on $\mu\left(\mathbb{C P}^{1}\right)$. For any twist

$$
t=\sum_{i<j} \frac{1}{2} \lambda_{i j} e_{i} \wedge e_{j} \in \Lambda^{2} \mathfrak{g}\left(\lambda_{i j} \in \mathbb{R}\right),
$$

we obtain

$$
\mathfrak{g}_{t}^{*}=\operatorname{span}\left\{\varepsilon^{1}+\lambda_{12} e_{2}+\lambda_{13} e_{3}, \varepsilon^{2}-\lambda_{12} e_{1}+\lambda_{13} e_{3}, \varepsilon^{3}-\lambda_{13} e_{1}-\lambda_{23} e_{2}\right\} .
$$

We calculate as

$$
\begin{aligned}
\left(\varepsilon^{1}+\lambda_{12} e_{2}+\lambda_{13} e_{3}\right)_{\mathfrak{g}^{*}} & =-\left(1+2 \lambda_{12} \xi_{3}-2 \lambda_{13} \xi_{2}\right) \frac{\partial}{\partial \xi_{1}}-2 \lambda_{13} \xi_{1} \frac{\partial}{\partial \xi_{2}}+2 \lambda_{12} \xi_{1} \frac{\partial}{\partial \xi_{3}} \\
\left(\varepsilon^{2}-\lambda_{12} e_{1}+\lambda_{13} e_{3}\right)_{\mathfrak{g}^{*}} & =2 \lambda_{23} \xi_{2} \frac{\partial}{\partial \xi_{1}}-\left(1+2 \lambda_{12} \xi_{3}+2 \lambda_{23} \xi_{1}\right) \frac{\partial}{\partial \xi_{2}}+2 \lambda_{12} \xi_{2} \frac{\partial}{\partial \xi_{3}} \\
\left(\varepsilon^{3}-\lambda_{13} e_{1}-\lambda_{23} e_{2}\right)_{\mathfrak{g}^{*}} & =2 \lambda_{23} \xi_{3} \frac{\partial}{\partial \xi_{1}}-2 \lambda_{13} \xi_{3} \frac{\partial}{\partial \xi_{2}}-\left(1-2 \lambda_{13} \xi_{2}+2 \lambda_{23} \xi_{1}\right) \frac{\partial}{\partial \xi_{3}} .
\end{aligned}
$$

Then $\mathfrak{g}_{t}^{*}$ is admissible at $\xi=\left(\xi_{1}, \xi_{2}, \xi_{3}\right)$ in $\mathfrak{g}^{*}$ if and only if the matrix

$$
A_{t}(\xi)=\left(\begin{array}{ccc}
1+2 \lambda_{12} \xi_{3}-2 \lambda_{13} \xi_{2} & 2 \lambda_{13} \xi_{1} & -2 \lambda_{12} \xi_{1} \\
-2 \lambda_{23} \xi_{2} & 1+2 \lambda_{12} \xi_{3}+2 \lambda_{23} \xi_{1} & -2 \lambda_{12} \xi_{2} \\
-2 \lambda_{23} \xi_{3} & 2 \lambda_{13} \xi_{3} & 1-2 \lambda_{13} \xi_{2}+2 \lambda_{23} \xi_{1}
\end{array}\right)
$$

is regular. By computing the determinant of the matrix, we have

$$
f_{t}(\xi)=\operatorname{det} A_{t}(\xi)=\left(1+2 \lambda_{23} \xi_{1}-2 \lambda_{13} \xi_{2}+2 \lambda_{12} \xi_{3}\right)^{2} .
$$

So the complement $\mathfrak{g}_{t}^{*}$ is admissible at $\xi=\left(\xi_{1}, \xi_{2}, \xi_{3}\right)$ if and only if $1+$ $2 \lambda_{23} \xi_{1}-2 \lambda_{13} \xi_{2}+2 \lambda_{12} \xi_{3} \neq 0$.

Therefore $\mathfrak{g}_{t}^{*}$ is admissible on $\mu\left(\mathbb{C P}^{1}\right)$ if and only if the "non-admissible surface" $\left\{\xi=\left(\xi_{1}, \xi_{2}, \xi_{3}\right) \in \mathfrak{g}^{*} \mid 1+2 \lambda_{23} \xi_{1}-2 \lambda_{13} \xi_{2}+2 \lambda_{12} \xi_{3} \neq 0\right\}$ for $\mathfrak{g}_{t}^{*}$ and the image $\mu\left(\mathbb{C P}^{1}\right)$ have no common point. Since $\mu\left(\mathbb{C P}^{1}\right)$ is the 2 -sphere with center at the origin and with radius $\frac{1}{2}$, we can see that this condition is equivalent to the condition

$$
\lambda_{12}^{2}+\lambda_{13}^{2}+\lambda_{23}^{2}<1
$$

From the above disscusion, we obtain the following theorem. 
Theorem 4.1. If a twist $t:=\sum_{i<j} \frac{1}{2} \lambda_{i j} e_{i} \wedge e_{j}$ satisfies $\lambda_{12}^{2}+\lambda_{13}^{2}+\lambda_{23}^{2}<1$, then the Fubini-Study form $\omega_{\mathrm{FS}}$ on $\mathbb{C P}^{1}$ can be deformed by $t$ in the sense of Section 3 .

We shall see an example of twists giving symplectomorphisms on $\mathbb{C P}^{1}$.

Example 3. We use a twist $t=\frac{1}{2} X_{12} \wedge Y_{12}$ in $\Lambda^{2} \mathfrak{s u}(2)$ and a real number $\lambda$, where $-1<\lambda<1$. The symplectic structure $\omega_{\mathrm{FS}}^{\lambda t}$ deformed $\omega_{\mathrm{FS}}$ by $\lambda t$ is written by

$$
\omega_{\mathrm{FS}}^{\lambda t}=\left\{\left(1+\frac{1}{2} \lambda\right)\left(x_{1}^{2}+y_{1}^{2}\right)^{2}+2\left(x_{1}^{2}+y_{1}^{2}\right)+\left(1-\frac{1}{2} \lambda\right)\right\}^{-1} d x_{1} \wedge d y_{1}
$$

on $U_{1}$. Then it follows from an elementary calculation that the symplectic volume $\operatorname{Vol}\left(\mathbb{C P}^{1}, \omega_{\mathrm{FS}}^{\lambda t}\right)$ of $\left(\mathbb{C P}^{1}, \omega_{\mathrm{FS}}^{\lambda t}\right)$ is

$$
\operatorname{Vol}\left(\mathbb{C P}^{1}, \omega_{\mathrm{FS}}^{\lambda t}\right)= \begin{cases}\pi & (\lambda=0) \\ \frac{\pi}{\lambda} \log \left|\frac{2+\lambda}{2-\lambda}\right| & (\lambda \neq 0) .\end{cases}
$$

Next, we consider a cohomology class of each $\omega_{\mathrm{FS}}^{\lambda t}$. Since $H_{\mathrm{DR}}^{2}\left(\mathbb{C P}^{1}\right)=\mathbb{R}$, there exists a real number $k_{\lambda} \in \mathbb{R}$ such that $\left[\omega_{\mathrm{FS}}^{\lambda t}\right]=k_{\lambda}\left[\omega_{\mathrm{FS}}\right]$. By integrating, we obtain

$$
k_{\lambda}=\frac{1}{\lambda} \log \left|\frac{2+\lambda}{2-\lambda}\right|,
$$

where $\lambda \neq 0$. Since the function $k_{\lambda}$ of $\lambda$ is smooth, two symplectic structures $\omega_{\mathrm{FS}}$ and $k_{\lambda} \omega_{\mathrm{FS}}^{\lambda t}$ are cohomologous. In particular, $\left(\mathbb{C P}^{1}, \omega_{\mathrm{FS}}\right)$ and $\left(\mathbb{C P}^{1}, k_{\lambda} \omega_{\mathrm{FS}}^{\lambda t}\right)$ are symplectomorphic by Moser's theorem.

Next we shall see deformations of symplectic structures in the case of $\mathbb{C P}^{n}$.

Example 4. We consider the case of $n=2$. Since $\left[Y_{23}, 2 Z_{1}-Z_{2}\right]=0$ in $\Lambda^{2} \mathfrak{s u}(3)$, we use the twist $t=\frac{1}{2} \lambda Y_{23} \wedge\left(2 Z_{1}-Z_{2}\right)(\lambda \in \mathbb{R})$ to deform $\omega_{\mathrm{FS}}$. Then $\omega_{\mathrm{FS}}$ is deformed to

$$
\begin{aligned}
& \omega_{F S}^{t}=\omega_{F S}+ \frac{\lambda}{\left\{\sum_{k}\left(x_{k}^{2}+y_{k}^{2}\right)+1\right\}^{3}}\left\{\left(x_{1} y_{2}-x_{2} y_{1}\right) d x_{1} \wedge d y_{1}\right. \\
&+\left(x_{1}^{2}-x_{2}^{2}\right) d x_{1} \wedge d x_{2}+\left(x_{1} y_{1}-x_{2} y_{2}\right) d x_{1} \wedge d y_{2} \\
& \quad+\left(x_{1} y_{1}-x_{2} y_{2}\right) d y_{1} \wedge d x_{2}+\left(y_{1}^{2}-y_{2}^{2}\right) d y_{1} \wedge d y_{2} \\
&\left.\quad-\left(x_{1} y_{2}-x_{2} y_{1}\right) d x_{2} \wedge d y_{2}\right\}
\end{aligned}
$$

on $U_{1}$, where $x_{i}:=\operatorname{Re} \frac{z_{i+1}}{z_{1}}$ and $y_{i}:=\operatorname{Im} \frac{z_{i+1}}{z_{1}}$. 
Example 5. The next example is a symplectic toric manifold $\mathbb{C P}^{n}$ with the torus action:

$$
\left(e^{i \theta_{2}}, e^{i \theta_{3}}, \ldots, e^{i \theta_{n+1}}\right) \cdot\left[z_{1}: \cdots: z_{n+1}\right]:=\left[z_{1}: e^{i \theta_{2}} z_{2}: \cdots: e^{i \theta_{n+1}} z_{n+1}\right]
$$

for any $\theta_{i}$ in $\mathbb{R}$. The moment map $\mu: \mathbb{C P}^{n} \rightarrow \mathbb{R}^{n}$ for this action on $\left(\mathbb{C P}^{n}, \omega_{\mathrm{FS}}\right)$ is

$$
\mu\left(\left[z_{1}: \cdots: z_{n+1}\right]\right):=-\frac{1}{2}\left(\frac{\left|z_{2}\right|^{2}}{|z|^{2}}, \ldots, \frac{\left|z_{n+1}\right|^{2}}{|z|^{2}}\right),
$$

where $z=\left(z_{1}, \ldots, z_{n+1}\right)$ in $\mathbb{C}^{n}$. We set $X_{1}:=(1,0, \ldots, 0), \ldots, X_{n}:=$ $(0, \ldots, 0,1)$. Since $\mathbb{T}^{n}$ is commutative, the brackets $\left[X_{i}, X_{j}\right]$ vanish for all $i$ and $j$. Hence for any $\lambda_{12}$ in $\mathbb{R}$, the twist $t_{12}:=\lambda_{12} X_{1} \wedge X_{2}$ deforms $\omega_{\mathrm{FS}}$ to a symplectic structure $\omega_{\mathrm{FS}}^{t_{12}}$ induced by a Poisson structure $\pi_{\mathrm{FS}}^{t_{12}}:=\pi_{\mathrm{FS}}-\left(t_{12}\right)_{\mathbb{C P}^{n}}$ by Theorem 3.2. On the other hand it follows $\pi_{\mathbb{T}^{n}}^{t}=t^{L}-t^{R}=0$ for any twist $t$ by the commutativity of $\mathbb{T}^{n}$. Therefore, after deformation, the multiplicative Poisson structure 0 on $\mathbb{T}^{n}$ is invariant and this action is a symplectic-Hamiltonian action with the same moment map $\mu$. Therefore, by Theorem 3.2 again, the twist $t_{13}:=\lambda_{13} X_{1} \wedge X_{3}$ deforms $\omega_{\mathrm{FS}}^{t_{12}}$ to $\left(\omega_{\mathrm{FS}}^{t_{12}}\right)^{t_{13}}=\omega_{\mathrm{FS}}^{t_{12}+t_{13}}$ induced by $\left(\pi_{\mathrm{FS}}^{t_{12}}\right)^{t_{13}}=\pi_{\mathrm{FS}}^{t_{12}+t_{13}}$. Then we see that the trivial Poisson structure on $\mathbb{T}^{n}$ is invariant and that the action is a symplectic-Hamiltonian action with $\mu$. By repeating this operation, it follows that we can deform $\omega_{\mathrm{FS}}$ to $\omega_{\mathrm{FS}}^{t}$ for any twist $t=\sum_{i<j} \lambda_{i j} X_{i} \wedge X_{j}$ and that the action is a symplectic-Hamiltonian action with $\mu$. On $U_{1}$, since we obtain

$$
\begin{aligned}
& \left(X_{i} \wedge X_{j}\right)_{\mathbb{C P}^{n}}=y_{i} y_{j} \frac{\partial}{\partial x_{i}} \wedge \frac{\partial}{\partial x_{j}}-y_{i} x_{j} \frac{\partial}{\partial x_{i}} \wedge \frac{\partial}{\partial y_{j}}-x_{i} y_{j} \frac{\partial}{\partial y_{i}} \wedge \frac{\partial}{\partial x_{j}}+x_{i} x_{j} \frac{\partial}{\partial y_{i}} \wedge \frac{\partial}{\partial y_{j}} \\
& (1 \leq i<j \leq n)
\end{aligned}
$$

where $x_{i}:=\operatorname{Re} \frac{z_{i+1}}{z_{1}}$ and $y_{i}:=\operatorname{Im} \frac{z_{i+1}}{z_{1}}$, it follows that

$$
\begin{aligned}
\omega_{\mathrm{FS}}^{t}=\omega_{\mathrm{FS}}+\sum_{i<j} \frac{\lambda_{i j}}{\left\{\sum_{k}\left(x_{k}^{2}+y_{k}^{2}\right)+1\right\}^{3}}\left(x_{i} x_{j} d x_{i} \wedge d x_{j}\right. \\
\left.\quad+x_{i} y_{j} d x_{i} \wedge d y_{j}+y_{i} x_{j} d y_{i} \wedge d x_{j}+y_{i} y_{j} d y_{i} \wedge d y_{j}\right) .
\end{aligned}
$$

The last example is the complex Grassmannian $\operatorname{Gr}\left(r ; \mathbb{C}^{n}\right):=\mathrm{SU}(n) /(\mathrm{S}(\mathrm{U}(r) \times$ $\mathrm{U}(n-r)))$ with the Kirillov-Kostant form $\omega_{\mathrm{KK}}$. With respect to $\omega_{\mathrm{KK}}$, the natural $\mathrm{SU}(n)$-action is symplectic-Hamiltonian ([9]). 
Then we consider the following r-matrix of $\mathfrak{s u}(n)$ :

$$
t=\frac{1}{4 n} \sum_{1 \leq i<j \leq n} X_{i j} \wedge Y_{i j},
$$

where the r-matrix $t$ is the canonical one defined on any compact semi-simple Lie algebra over $\mathbb{R}$ (for example, see [3]). This is an r-matrix such that $[t, t] \neq 0$. We show that it satisfies $[t, t]_{M}=0$, where $M:=\operatorname{Gr}\left(r ; \mathbb{C}^{n}\right)$. Since $t$ is an r-matrix, the element $[t, t]$ is ad-invariant by the definition. Therefore $[t, t]$ is Ad-invariant because $\mathrm{SU}(n)$ is connected. By the definition of the $\mathrm{SU}(n)$-action on $\operatorname{Gr}\left(r ; \mathbb{C}^{n}\right)$, it follows that

$$
[t, t]_{M}=p_{*}[t, t]^{R},
$$

where $p: \mathrm{SU}(n) \rightarrow \operatorname{Gr}\left(r ; \mathbb{C}^{n}\right)=\mathrm{SU}(n) /(\mathrm{S}(\mathrm{U}(r) \times \mathrm{U}(n-r)))$ is the natural projection. Since any point $m$ in $\operatorname{Gr}\left(r ; \mathbb{C}^{n}\right)$ is represented by $g H$, where $g$ in $\mathrm{SU}(n)$ and $H:=\mathrm{S}(\mathrm{U}(r) \times \mathrm{U}(n-r))$, we compute

$$
[t, t]_{M, m}=p_{*}[t, t]_{g}^{R}=p_{*} R_{g *}[t, t] .
$$

Because of the Ad-invariance of $[t, t]$, we obtain

$$
p_{*} R_{g *}[t, t]=p_{*} L_{g *} L_{g^{-1} *} R_{g *}[t, t]=p_{*} L_{g *} \operatorname{Ad}_{g^{-1}}[t, t]=p_{*} L_{g *}[t, t] .
$$

Let $\mathfrak{h}$ be the Lie algebra of $H$. For any $X$ in $\mathfrak{h}$ and $g$ in $\mathrm{SU}(n)$, we compute

$p_{*} L_{g *} X=\left.p_{*} L_{g *} \frac{d}{d s} \exp s X\right|_{s=0}=\left.\frac{d}{d s}(g \exp s X) H\right|_{s=0}=\left.\frac{d}{d s} g H\right|_{s=0}=0$,

where we have used that $\exp s X$ is in $H$ in the third equality. Therefore it holds that $[t, t]_{M}=0$ if each term of $[t, t]$ includes elements in $\mathfrak{h}$ as follows. We notice that

$\mathfrak{h}=\operatorname{span}_{\mathbb{R}}\left\{X_{i j}, Y_{i j}, Z_{k} \mid 1 \leq i<j \leq r\right.$ or $r+1 \leq i<j \leq n$, and $\left.k=1, \ldots, n-1\right\}$

If $X_{i j}, Y_{i j} \in \mathfrak{h}$, then

$$
\left[\cdot, X_{i j} \wedge Y_{i j}\right]=\left[\cdot, X_{i j}\right] \wedge Y_{i j}-X_{i j} \wedge\left[\cdot, Y_{i j}\right]
$$

So these terms include an element in $\mathfrak{h}$. Hence we investigate terms of the form

$$
\begin{aligned}
{\left[X_{i j} \wedge Y_{i j}, X_{k l} \wedge Y_{k l}\right]=\quad } & -\left[X_{i j}, X_{k l}\right] \wedge Y_{i j} \wedge Y_{k l}-X_{i j} \wedge\left[Y_{i j}, X_{k l}\right] \wedge Y_{k l} \\
& -Y_{i j} \wedge\left[X_{i j}, Y_{k l}\right] \wedge X_{k l}-X_{i j} \wedge X_{k l} \wedge\left[Y_{i j}, Y_{k l}\right]
\end{aligned}
$$


where $X_{i j}, Y_{i j}, X_{k l}$ and $Y_{k l}$ are not in $\mathfrak{h}$. In the case of $i=k$ and $j=l$, we get

$$
\begin{array}{r}
{\left[X_{i j}, X_{i j}\right]=\left[Y_{i j}, Y_{k l}\right]=0,} \\
{\left[X_{i j}, Y_{i j}\right]=2\left(Z_{i}-Z_{j}\right) \in \mathfrak{h},}
\end{array}
$$

where $Z_{n}=0$. In the case of $i=k$ and $j<l$ (resp. $l<j$ ), since it follows that $r+1 \leq j, l \leq n$, we obtain

$$
\begin{gathered}
{\left[X_{i j}, X_{k l}\right]=\left[Y_{i j}, Y_{k l}\right]=-X_{j l}\left(\operatorname{resp} . X_{l j}\right) \in \mathfrak{h},} \\
{\left[Y_{i j}, X_{k l}\right]=\left[Y_{k l}, X_{i j}\right]=-Y_{j l}\left(\operatorname{resp} . Y_{l j}\right) \in \mathfrak{h} .}
\end{gathered}
$$

We can also show the case of $i<k$ (resp. $k<i$ ) and $j=l$ in the similar way. Therefore all terms of $[t, t]$ include elements in $\mathfrak{h}$, so that $[t, t]_{M}=0$. Since $\operatorname{Gr}\left(r ; \mathbb{C}^{n}\right)$ is compact, for sufficiently small $|\lambda|$, a 2-vector field $\pi_{\mathrm{KK}}^{\lambda t}$ is nondegenerate by Theorem 3.1, where $\pi_{\mathrm{KK}}$ is the Poisson structure induced by $\omega_{\mathrm{KK}}$. Example 3 is the special case of this example. From the above discussion, we obtain the following.

Theorem 4.2. Let $t$ be the above r-matrix of $\mathfrak{s u}(n)$. Then there exists sufficiently small number $\lambda$ such that the Kirillov-Kostant form $\omega_{\mathrm{KK}}$ on $\operatorname{Gr}\left(r ; \mathbb{C}^{n}\right)$ can be deformed by a twist $\lambda t$ in the sense of Section 3 .

\section{acknowledgments}

I would like to express my deepest gratitude to Yuji Hirota for leading me into the study of quasi-Poisson theory and my supervisor Yasushi Homma for his helpful advice.

\section{References}

[1] A. Alekseev and Y. Kosmann-Schwarzbach. Manin pairs and moment maps. J. Diff. Geom. 56 (2000) 133-165.

[2] H. Bursztyn and M. Crainic. Dirac geometry, quasi-Poisson actions and $D / G$-valued moment maps. J. Diff. Geom. 823 (2009) 501-566.

[3] V. G. Drinfel'd. Hamiltonian structures on Lie groups, Lie bialgebras and the geometric meaning of the classical Yang-Baxter equations. Soviet Math. Dokl. 27 (1983) 68-71. 
[4] J. -H. Lu. Momentum mappings and reduction of Poisson actions, in Symplectic Geometry, Groupoids and Integrable Systems, P. Dazord and A. Weinstein, eds. Springer (1991) 209-226.

[5] J. -H. Lu. Multiplicative and Affine Poisson structures on Lie groups. Berkeley Thesis (1991).

[6] J. -H. Lu and A. Weinstein. Poisson-Lie groups, dressing transformations and Bruhat decompositions. J. Diff. Geom. 31 (1990) 501-526.

[7] J. E. Marsden and T. S. Ratiu. Introduction to Mechanics and Symmetry Second Edition. (Springer, 2003).

[8] D. Salamon. Uniqueness of Symplectic Structures. arXiv:1211.2940v5

[9] A. C. da Silva. Lectures on Symplectic Geometry. (Springer-Verlag, 2006).

[10] I. Vaisman. Lectures on the Geometry of Poisson Manifold. Birkhaeuser (1994). 